PRATISSOLI D; POLANCZYK A; PEREIRA CLT; FURTADO ISA; COCHETO JG. 2007. Influência da fase embrionária dos ovos da traça-das-crucíferas sobre fêmeas de Trichogramma pretiosum com diferentes idades. Horticultura Brasileira 25: 286-290.

\title{
Influência da fase embrionária dos ovos da traça-das-crucíferas sobre fêmeas de Trichogramma pretiosum com diferentes idades
}

\author{
Dirceu Pratissoli; Ricardo Antonio Polanczyk; Cácia Leila Tigre Pereira; Idana Soraya de Andrade \\ Furtado; Juliéder Goronci Cocheto \\ UFES, CCA, Depto. fitotecnia, Alto Universitário s/n, 29500-000 Alegre-ES; pratissoli@ cca.ufes.br
}

\begin{abstract}
RESUMO
Plutella xylostella é considerada a praga mais importante das crucíferas. O método de controle mais utilizado para essa praga é o químico. Contudo, esta espécie de inseto vem desenvolvendo resistência aos inseticidas aplicados. O controle biológico com espécies do gênero Trichogramma é considerado uma alternativa no controle dessa praga. Porém, poucos são os trabalhos que mencionam aspectos biológicos desse parasitóide sobre esta praga. Neste trabalho avaliou-se a influência da fase embrionária dos ovos do hospedeiro $P$. xylostella sobre fêmeas de $T$. pretiosum com diferentes idades. Fêmeas do parasitóide foram divididas em cinco lotes, compostos por espécimes recém-emergidos com 24; 48; 72 e 96 horas de idade. Cada lote continha dez fêmeas de T. pretiosum. Para cada fêmea de cada lote, foi oferecida uma cartela contendo 30 ovos de $P$. xylostella com um, dois e três dias de idade. As maiores taxas de parasitismo foram observadas em fêmeas com idade superior a 48 horas, independente do desenvolvimento embrionário do hospedeiro. Em ovos com três dias de desenvolvimento embrionário verificou-se que, para fêmeas recém-emergidas e com 48 horas de idade, a taxa de viabilidade foi superior apenas em relação àquelas com 96 horas de idade. Ao se avaliar os descendentes de T. pretiosum provenientes de ovos com um dia de desenvolvimento embrionário, verificou-se que os maiores valores de longevidade foram obtidos quando as fêmeas desse parasitóide eram recém-emergidas.
\end{abstract}

Palavras-chave: Plutella xylostella, repolho, controle biológico, parasitóide de ovos.

\begin{abstract}
Influence of diamondback moth embrionary egg stage on Trichogramma pretiosum females of different ages

Plutella xylostella is one of the most important pests of Cruciferae. Chemicals have been used to control this insect, but the rapid development of resistance is a serious constraint to this method. Biological control with Trichogramma species has been reported as an alternative to control diamondback moth. However few works report biological parameters of this parasitoid interacting with this pest. This work was carried out to evaluate the influence of egg embryonary stage of $P$. xylostella on $T$. pretiosum females at different ages. Females of this parasitoid were organized in five groups. These groups were composed of females just emerged, $24 \mathrm{~h} ; 48 \mathrm{~h} ; 72 \mathrm{~h}$, and 96 hours-old. Each group was formed of ten females. Each female received a card with thirty $P$. xylostella eggs, one, two and three day-old. Higher parasitism rates were observed with females older than 48 hours, with no influence of host embryonic development. In three day-old eggs it was verified that just emerged females and 48 hour-old, the viability index was superior just to those 96 hour-old. The progeny with one day of embryonary development, presented higher longevity values with just emerged females.
\end{abstract}

Keywords: Plutella xylostella, cabbage, biological control, egg parasitoid.

(Recebido para publicação em 18 de julho de 2006; aceito em 14 de abril de 2007)

\begin{abstract}
A traça-das-crucíferas Plutella xylostella (L.) (Lepidoptera: Plutellidae), é considerada a praga mais importante das crucíferas em escala mundial, ocasionando grandes perdas em plantios comerciais (França \& Medeiros, 1998; Castelo Branco \& Gatehouse, 2001). O principal método de controle para essa praga tem sido a utilização cada vez maior de inseticidas, o que vem propiciando o desenvolvimento de resistência a esses produtos e até mesmo a produtos biológicos como os produzidos a partir de Bacillus thuringiensis Berliner (Perez et al., 1997).

O controle biológico de $P$. xylostella, quando bem implantado, pode ser uma excelente alternativa frente às habituais recomendações de controle químico

(Krnjajic et al., 1997). O elevado número de trabalhos mencionando o complexo de parasitóides nas diferentes regiões produtoras de crucíferas demonstra a importância desses inimigos naturais para a manutenção do nível populacional dessa praga abaixo do nível de dano econômico (Mitchel et al., 1998).

As espécies de parasitóides pertencentes ao gênero Trichogramma têm sido muito estudadas e utilizadas em programas de controle biológico, fato esse atribuído à sua eficiência, ampla distribuição geográfica, facilidade de criação massal em ovos de hospedeiros alternativos, com menor custo de produção, permitindo sua utilização em liberações inundativas, além desse parasitóide já ter sido coletado em mais
\end{abstract}

de 200 hospedeiros, pertencentes a mais de 70 famílias e oito ordens de insetos (Zucchi \& Monteiro, 1997).

Várias espécies de Trichogramma têm sido mencionadas como eficientes em relação ao seu potencial de uso no controle de P. xylostella em diversos países, como T. ostriniae Pang \& Chen, T. chilonis Ishii e T. pintoi Voegelé na Alemanha (Wuhrer \& Hassan, 1993); T. pretiosum e $T$. minutum Riley nos EUA (Vasquez et al., 1997); T. voegelei Pintureau, T. oleae Voegelé \& Pointel, T. dendrolimi Matsumura, T. exigum Pinto \& Platner, T. chilonis, T. pretiosum, T. buesi Voegelé, T. ostrinae e Trichogramatoidea bactrae Nagaraja na França (Tabone et al., 1999).

Aspectos biológicos de $T$. pretiosum já foram pesquisados em diferentes 
hospedeiros e temperaturas (Basso et al., 1998; Barros \& Vendramim, 1999). Contudo, no Brasil, são escassos os relatos de pesquisas mencionando aspectos biológicos desse parasitóide quando criado em ovos de P. xylostella. Segundo Oliveira et al. (2003), um dos fatores que podem ser responsáveis pelo sucesso ou fracasso da utilização de parasitóides do gênero Trichogramma no controle de lepidópteros-praga é o conhecimento de parâmetros biológicos deste parasitóide quando associado a determinado hospedeiro alvo e à fase embrionária dos ovos desse hospedeiro.

Assim, o estudo das características biológicas de $T$. pretiosum em função do hospedeiro e da fase embrionária dos ovos pode fornecer informações importantes para a implantação de programas de manejo integrado de $P$. xylostella. $\mathrm{O}$ objetivo desta pesquisa foi obter informações básicas sobre aspectos biológicos de $T$. pretiosum, de diferentes idades, criado em ovos de P. xylostella de diferentes fases embrionárias.

\section{MATERIAL E MÉTODOS}

Criação de $P$. xylostella Inicialmente, cerca de 500 pupas de $P$. xylostella provenientes da criação estoque de laboratório da UFRPE foram transferidas para o laboratório de entomologia da UFES, onde foi iniciada a criação da traça-das-crucíferas em folhas de couve, segundo método adotado por Barros \& Vendramim (1999).

Coleta, manutenção e multiplicação de $T$. pretiosum A espécie $T$. pretiosum, proveniente da criação estoque do laboratório de entomologia da UFES foi inicialmente coletada em plantios comerciais de tomate localizados no município de Alegre (ES). Para a coleta dessa espécie, foram utilizadas cartelas contendo ovos de Anagasta kuehniella (Zeller) inseridas em pequenas gaiolas fixas, por 48 horas, nas folhas do terço superior de plantas de tomate. Após a coleta, a espécie foi identificada pelo professor Roberto Antonio Zucchi (Depto. de Entomologia, Fitopatologia e Zoologia Agrícola, ESALQ/USP).

Para manutenção dos parasitóides, ovos de A. kuehniella obtidos segundo técnica adaptada de Parra (1997), foram

Tabela 1. Médias ( \pm erro padrão) da porcentagem de parasitismo de Trichogramma pretiosum recém-emergido e com 24, 48, 72 e 96 horas, em ovos de Plutella xylostella em diferentes fases de desenvolvimento embrionário $\left(T=25 \pm 1^{\circ} \mathrm{C}\right.$; $\mathrm{UR}=70 \pm 10 \%$ e fotofase $=14$ horas) (average $( \pm$ standard deviation) of the percentual of parasitism of just emerged Trichogramma pretiosum after 24, 48, 72 and 96 hours, on eggs of Plutella xylostella in different embrionary ages). Porto Alegra, UFES, 2005.

\begin{tabular}{|c|c|c|c|}
\hline \multirow{2}{*}{$\begin{array}{l}\text { Idade de } \mathrm{T} \text {. } \\
\text { pretiosum }\end{array}$} & \multicolumn{3}{|c|}{ Parasitismo (\%) } \\
\hline & 1 dia & 2 dias & 3 dias \\
\hline Recém-emergido & $35,0 \pm 3,42 \mathrm{cA}$ & $30,3 \pm 1,79 \mathrm{bA}$ & $33,3 \pm 1,94 \mathrm{bA}$ \\
\hline $24 \mathrm{~h}$ & $40,0 \pm 3,31 \mathrm{bA}$ & $36,3 \pm 1,45 \mathrm{bA}$ & $38,3 \pm 1,84 a A$ \\
\hline $48 \mathrm{~h}$ & $62,7 \pm 3,77 \mathrm{aA}$ & $50,3 \pm 3,75 a B$ & $40,0 \pm 1,70 \mathrm{aC}$ \\
\hline $72 \mathrm{~h}$ & $58,3 \pm 2,01 \mathrm{aA}$ & $43,4 \pm 1,40 a B$ & $36,7 \pm 1,15 \mathrm{aB}$ \\
\hline $96 \mathrm{~h}$ & $60,7 \pm 2,86 \mathrm{aA}$ & $59,7 \pm 2,56 \mathrm{aA}$ & $41,6 \pm 1,65 \mathrm{aB}$ \\
\hline
\end{tabular}

Médias seguidas pela mesma letra maiúscula na linha e minúscula na coluna não diferem entre si pelo teste de Tukey a 5\% de probabilidade (Average values followed by the same letter, large in the line and small in the collumn did not differ through the Tukey test, 5\%).

inviabilizados e fixados com goma arábica diluída a $30 \%$ em cartelas $(8 \times 2$ $\mathrm{cm})$ de cartolina azul celeste. Estas foram oferecidas para adultos recémemergidos em tubos de vidro $(8,5 \times 2,4)$ e mantidos em câmaras climatizadas, com temperatura de $25 \pm 1^{\circ} \mathrm{C}$, umidade relativa de $70 \pm 10 \%$ e fotofase de 14 horas.

Influência da fase embrionária dos ovos de $P$. xylostella sobre fêmeas de $T$. pretiosum Fêmeas de T. pretiosum foram divididas em cinco lotes, compostos por fêmeas recém-emergidas e com 24; 48; 72 e 96 horas de idade. Cada lote representava, além da idade dos parasitóides, os diferentes períodos de ausência do hospedeiro sendo composto por dez fêmeas. Cada fêmea do parasitóide foi acondicionada em tubos de vidro $(3 \times 0,5 \mathrm{~cm})$, fechados com filme plástico do tipo PVC e que continham na parede interna gotículas de mel para alimentação.

Trinta ovos de P. xylostella, com um, dois e três dias de idade, foram coletados de discos de folha de couve (Brassica oleracea var. acephala DC, cultivar Manteiga) da criação em laboratório e transferidos com auxílio de um pincel de pêlos finos umedecidos para cartelas de cartolina azul celeste $(2,5 \times 0,3 \mathrm{~cm})$, as quais foram posteriormente colocadas nos tubos de vidro com uma fêmea do parasitóide e fechados com filme plástico PVC. Ao final de 24 horas de parasitismo, as fêmeas de $T$. pretiosum foram retiradas e descartadas, os tubos com as cartelas, foram mantidos em câmaras climatizadas, reguladas na temperatura de $25 \pm 1^{\circ} \mathrm{C}$, umidade relativa de $70 \pm 10 \%$ e fotofase de 14 horas, até a emergência dos descendentes. Foram avaliadas a porcentagem de parasitismo, a emergência dos parasitóides e a longevidade dos descendentes fêmeas, por meio da individualização de 30 fêmeas recém-emergidas e observações diárias para contagem da mortalidade.

$\mathrm{O}$ delineamento experimental foi inteiramente ao acaso, em esquema fatorial 3 x 5 (três: idades de ovos; cinco: idades do parasitóide), com dez repetições para cada combinação de níveis. Os dados foram submetidos à análise de variância e as médias comparadas pelo teste de Tukey (Pd"0,05), utilizando o programa computacional SAEG (Versão 5.0).

\section{RESULTADOS E DISCUSSÃO}

A porcentagem de parasitismo não diferiu para fêmeas de T. pretiosum com até 24 horas de idade nas diferentes fases embrionárias dos ovos de $P$. xylostella (Tabela 1). Contudo, a partir das 48 horas de idade a porcentagem de parasitismo foi maior em ovos com um dia de desenvolvimento embrionário, exceto para as fêmeas com 96 horas de idade, que apresentaram maior parasitismo em ovos com um e dois dias de desenvolvimento embrionário.

Com relação à idade de $T$. pretiosum, observou-se que o percentual de parasitismo foi maior em fêmeas com idade superior a 48 horas em ovos de $P$. xylostella com desenvolvimento embrionário de um e dois dias. Em ovos 
Tabela 2. Médias ( \pm erro padrão) da viabilidade (\%) de Trichogramma pretiosum recém emergido e com 24, 48, 72 e 96 horas, em ovos de Plutella xylostella em diferentes fases de desenvolvimento embrionário $\left(\mathrm{T}=25 \pm 1^{\circ} \mathrm{C} ; \mathrm{UR}=70 \pm 10 \%\right.$ e fotofase $=14$ horas) (Means ( \pm average value) of viability (\%) of just emerged Trichogramma pretiosum after 24, 48, 72 and 96 hours, on eggs of Plutella xylostella in different embrionary ages). Porto Alegre, UFES, 2005.

\begin{tabular}{lccc}
\hline $\begin{array}{l}\text { Idade de T. } \\
\text { pretiosum }\end{array}$ & \multicolumn{3}{c}{ Viabilidade (\%) } \\
\cline { 2 - 4 } & $98,3 \pm 1,14 \mathrm{aA}$ & $91,8 \pm 2,93 \mathrm{aA}$ & $96,4 \pm 1,41 \mathrm{aA}$ \\
Recém-emergido & $95,9 \pm 1,62 \mathrm{aA}$ & $97,3 \pm 1,82 \mathrm{aA}$ & $93,7 \pm 2,18 \mathrm{abA}$ \\
$\mathrm{h}$ & $94,5 \pm 1,71 \mathrm{aA}$ & $96,6 \pm 1,38 \mathrm{aA}$ & $96,6 \pm 1,85 \mathrm{aA}$ \\
$72 \mathrm{~h}$ & $95,8 \pm 1,76 \mathrm{aA}$ & $96,0 \pm 2,10 \mathrm{aA}$ & $95,4 \pm 1,80 \mathrm{abA}$ \\
$96 \mathrm{~h}$ & $97,9 \pm 1,05 \mathrm{aA}$ & $96,6 \pm 1,43 \mathrm{aA}$ & $88,3 \pm 3,65 \mathrm{bB}$ \\
\hline
\end{tabular}

Médias seguidas pela mesma letra maiúscula na linha e minúscula na coluna não diferem entre si pelo teste de Tukey a 5\% de probabilidade (Means ( \pm average value) followed by the same letter, large in the line and small in the collumn did not differ through the Tukey test, 5\%).

com três dias de idade, verificou-se que apenas fêmeas recém-emergidas apresentaram uma redução significativa no parasitismo.

O conhecimento dos parâmetros biológicos do parasitóide associado a determinado hospedeiro alvo é um dos principais fatores que implica no sucesso do controle biológico, sendo que a fase embrionária dos ovos deste hospedeiro pode também influenciar no comportamento do Trichogramma reduzindo a aceitação do hospedeiro e, conseqüentemente, a taxa de parasitismo (Pak, 1988; Van Dijken et al., 1986; Pereira et al., 2004). A taxa de parasitismo de T. pretiosum foi maior à medida que ocorreu aumento na idade das fêmeas, independente do desenvolvimento embrionário do ovo de P. xylostella. Resultado semelhante foi encontrado por Oliveira (1997) em fêmeas recémemergidas de Trichogramma maxacalii sobre ovos de A. kuehniella; contudo, quando esse autor trabalhou com a segunda geração de $T$. maxacalii sobre ovos do mesmo hospedeiro, observouse resultado semelhante aos encontrados por Berti \& Marcano (1991) e Fleury \& Bouletreau (1993), que constataram maiores taxas de parasitismo nas primeiras horas de vida do parasitóide. Estes resultados demonstram que o parasitismo por Trichogramma é influenciado pela idade e espécie do parasitóide e seu hospedeiro.

Fêmeas de $T$. pretiosum privadas do hospedeiro $P$. xylostella por até 24 horas não apresentaram diferenças nas taxas de parasitismo com o incremento do desenvolvimento embrionário do hos- pedeiro. Oliveira et al. (2003) relataram comportamento semelhante em fêmeas recém-emergidas de $T$. maxacalii em ovos de diferentes estágios de desenvolvimento embrionário de Oxydia vesulia (Cramer). Entretanto, fêmeas de $T$. pretiosum com 48 horas de idade apresentaram maior parasitismo em ovos de P. xylostella com um dia de desenvolvimento embrionário. $\mathrm{O}$ decréscimo do parasitismo provavelmente ocorre devido a mudanças nas características internas do ovo, pois com avanço do desenvolvimento embrionário os valores nutricionais vão sendo reduzidos, e este fato influencia no comportamento de aceitação do hospedeiro pelo parasitóide (Navarajan, 1979; Schmidt \& Smith, 1987). Resultados semelhantes foram relatados por Lopes \& Parra (1991), que observaram que o parasitismo de Trichogramma decresceu quando os ovos de Diatraea saccharalis (Frabr.) estavam no $5^{\circ}$ dia, época em que as lagartas estavam próximas da eclosão. Faria et al. (2000) também relataram que o parasitismo de T. pretiosum sobre ovos de Tuta absoluta (Meyrick) apresentou decréscimo à medida que a fase embrionária dos ovos avançava. Assim, a fase embrionária do hospedeiro parece ter grande influência sobre as características biológicas em Trichogramma.

Com relação à viabilidade, fêmeas com 96 horas de idade tiveram este parâmetro reduzido em ovos com três dias quando comparado aos ovos de um e dois dias (Tabela 2). Resultados semelhantes foram encontrados por Oliveira (1997) e Oliveira et al. (2003) com T. maxacalii e Trichogramma acacioi
Brun, Moraes \& Soares sobre ovos de A. kuehniella e $O$. vesulia, respectivamente. Lopes \& Parra (1991), avaliando a viabilidade de fêmeas recémemergidas de Trichogramma distinctum Zucchi em ovos de A. kuehniella, também observaram que não houve influência da fase embrionária do hospedeiro nesse parâmetro. A razão sexual de $T$. pretiosum foi igual a um, não sendo influenciada pelos tratamentos avaliados. Esta característica de obtenção de apenas fêmeas é desejável em criações de laboratório e pode ser atribuída a uma estabilidade genética, que ocorre com o aumento do número de gerações (Pereira et al., 2004).

A longevidade dos descendentes de T. pretiosum foi influenciada pela idade do parasitóide e pelas diferentes fases embrionárias dos ovos de $P$. xylostella (Tabela 3). Ao se analisar a fase embrionária dos ovos verificou-se que, em ovos com um dia de idade, a longevidade foi superior em relação às demais quando as fêmeas desse parasitóide eram recémemergidas. Em fêmeas com idade de 24 horas, a longevidade dos descendentes foi semelhante nas diferentes fases dos ovos de P. xylostella. Contudo, fêmeas de $T$. pretiosum com 48 e 72 horas de idade apresentaram maior longevidade dos descendentes em ovos com um e dois dias de desenvolvimento embrionário, enquanto as fêmeas do parasitóide com 96 horas de idade apresentaram maior longevidade de descendentes em ovos com dois dias de desenvolvimento embrionário.

A longevidade dos descendentes de T. pretiosum em ovos com um dia de desenvolvimento embrionário foi maior quando as fêmeas desse parasitóide eram recém-emergidas. Em ovos com dois dias de desenvolvimento embrionário constatou-se que a longevidade dos descendentes de $T$. pretiosum foi maior em fêmeas desse parasitóide com 48; 72 e 96 horas de idade. Quando os ovos de $P$. xylostella apresentavam três dias de desenvolvimento embrionário, a longevidade dos descendentes de $T$. pretiosum foi maior para as fêmeas desse parasitóide com 96 horas de idade. Valores semelhantes foram encontrados por Oliveira et al. (2003) em T. acacioi sobre ovos de $O$. vesulia. Contudo, Bai 
et al. (1995) constataram longevidades maiores trabalhando com outros hospedeiros e várias espécies de Trichogramma. A maior longevidade em parasitóides implica em indivíduos com tempo maior para procurar e parasitar ovos dos seus hospedeiros em campo, o que contribui para o aumento da sua população e da eficácia no controle de surtos posteriores de pragas.

Espécies do gênero Trichogramma têm sido amplamente estudadas em diversos países por serem altamente especializadas e eficientes, apresentando resultados promissores no controle de pragas das hortaliças, como $P$. xylostella (Alam, 1990; Hirashina et al., 1990; Cordeiro \& Cave, 1992; Pratissoli et al., 2004a). Contudo, Lopes (1988) relata que a idade do parasitóide pode influenciar tanto na longevidade como no parasitismo desse inimigo natural. De acordo com Berti \& Marcano (1991) e Pratissoli et al. (2004b), o isolamento de fêmeas de $T$. pretiosum sem ovos do hospedeiro por um determinado período faz com que o parasitismo decresça significativamente.

O uso de parasitóides do gênero Trichogramma no controle da traça $P$. xylostella tem sido importante em cultivos de repolho, pois esse inimigo natural atua em uma fase do ciclo biológico da praga incapaz de causar dano à cultura, sendo o Brasil o único país da América do Sul onde se tem relato de $T$. pretiosum parasitando ovos de $P$. xylostella (Barros \& Vendramim, 1999). Neste trabalho observou-se que as diferentes idades de fêmeas de T. pretiosum e as diferentes fases de desenvolvimento embrionário de $P$. xylostella afetam a capacidade de parasitismo e a longevidade dos descendentes desta espécie de parasitóide. Isto demonstra a importância da idade do parasitóide bem como o período de desenvolvimento embrionário do hospedeiro para manter a qualidade de $T$. pretiosum em laboratório, em uma criação massal, bem como nas liberações de campo para o controle biológico de pragas.

\section{AGRADECIMENTOS}

Ao CNPq pela concessão de bolsas.

Tabela 3. Médias ( \pm erro padrão) da longevidade dos descendentes (dias) de Trichogramma pretiosum recém-emergido e com 24, 48, 72 e 96 horas, em ovos de Plutella xylostella com diferentes fases de desenvolvimento embrionário $\left(\mathrm{T}=25 \pm 1^{\circ} \mathrm{C}\right.$; UR $=70 \pm 10 \%$ e fotofase $=$ 14 horas) (Means ( \pm average value) followed by the same letter, large in the line and small in the collumn did not differ through the Tukey test, 5\%). Porto Alegre, UFES, 2005.

\begin{tabular}{lccc}
\hline Idade de T. & \multicolumn{3}{c}{ Longevidade } \\
\cline { 2 - 4 } pretiosum (horas) & $\mathbf{1 ~ d i a}$ & $\mathbf{2 ~ d i a s}$ & $\mathbf{3}$ dias \\
\hline Recém-emergido & $1,7 \pm 0,09 \mathrm{aB}$ & $1,1 \pm 0,08 \mathrm{bB}$ & $1,0 \pm 0,06 \mathrm{bB}$ \\
24 & $1,1 \pm 0,06 \mathrm{bcA}$ & $1,0 \pm 0,03 \mathrm{bA}$ & $1,0 \pm 0,03 \mathrm{bA}$ \\
48 & $1,3 \pm 0,08 \mathrm{bcA}$ & $1,5 \pm 0,08 \mathrm{aA}$ & $1,1 \pm 0,05 \mathrm{bB}$ \\
72 & $1,4 \pm 0,00 \mathrm{bA}$ & $1,4 \pm 0,12 \mathrm{aA}$ & $1,0 \pm 0,03 \mathrm{bB}$ \\
96 & $1,1 \pm 0,08 \mathrm{cC}$ & $1,8 \pm 0,05 \mathrm{aA}$ & $1,4 \pm 0,10 \mathrm{aB}$ \\
\hline
\end{tabular}

Médias seguidas pela mesma letra maiúscula na linha e minúscula na coluna não diferem entre si pelo teste de Tukey a 5\% de probabilidade (Average values followed by the same letter, large in the line and small in the collumn did not differ through the Tukey test, 5\%)..

\section{REFERÊNCIAS}

ALAM MM. 1990. Diamondback moth and its natural enemies in Jamaica and some other Caribbean Islands. In: INTERNATIONAL WORKSHOOP OF DIAMONDBACK MOTH AND OTHER CRUCIFERS PESTS, 2., 1990, Taiwan. Proceeding... Taiwan: Asian Vegetable Research and Development Center. p.233-243.

BAI B; ÇOBANOGLU S; SMITH SM. 1995. Assessment of Trichogramma species for biological control of forest lepidopteran defoliators. Entomolgia Experimentalis et Applicat 75: 135-143.

BARROS R; VENDRAMIM JD. 1999. Efeito de cultivares de repolho, utilizados para criação de Plutella xylostella (L.) (Lepidoptera: Plutelidae), no desenvolvimento de Trichogramma pretiosum Riley (Hymenoptera: Trichogrammatidae). Anais da Sociedade Entomológica do Brasil 28: 469-476.

BASSO C; GRILLE G; POMPANON F; ALLEMAND R; PINTUREAU B. 1998. Comparación de los caracteres biológicos y etológicos Trichogramma pretiosum y de $T$. exiguum (Hymenoptera: Trichogrammatidae). Revista Chilena de Entomologia 25:45-53.

BERTI J; MARCANO R. 1991. Effect of time of host absence on parasitism by Trichogramma pretiosum Riley (Himenoptera: Trichogrammatidae). Boletin de Entomologia Venezolana 6: 5-10.

CASTELO BRANCO M; GATEHOUSE A. 2001. Survey of insecticide susceptibility in Plutella xylostella (L) (Lepidoptera: Yponomeutidae) in the Federal District, Brazil. Neotropical Entomololgy 30: 327-332.

CORDEIRO J; CAVE RD. 1992. Natural enemies of Plutella xylostella (Lep.: Plutellidae) on crucifers in Honduras. Entomophaga 37: 397-407.

FARIA CA; TORRES JB; FARIAS AMI. 2000. Resposta funcional de Trichogramma pretiosum Riley (Hymenoptera: Trichogrammatidae) parasitando ovos de Tuta absoluta (Meyrick) (Lepidoptera: Gelechiidae): efeito da idade do hospedeiro. Anais da Sociedade Entomológica do Brasil 29: 85-93.
FLEURY F; BOULETREAU M. 1993. Effects of temporary host deprivation on the reproductive potencial of Trichogramma brassicae. Entomologia Experimentalis et Applicata 68: 203-210.

FRANÇA FH; MEDEIROS MA. 1998. Impacto da combinação de inseticidas sobre a produção de repolho e parasitóides associados com a traça-das-crucíferas. Horticultura Brasileira 16: 132-135.

HIRASHIMA Y; MIURA K; MIURA T; SHIRO K. 1990. Studies on the biological control of the Diamondback Moth, Plutella xylostella (Linnaeus). II. Effect of temperature on the development of the egg parasitoids Trichogramma chilonis and Trichogramma ostriniae. Sci. Bull. Fac. Agric. 44: 65-70.

KRNJAJIC S; DIMIC N; PERIC P; VURSA M; CVETKOVIC M. 1997. Biological control of cabbage pests. Acta Horticulturae 462: 119124.

LOPES JRS. 1988. Estudos bioetológicos de Trichogramma galloi Zucchi, 1988 (Hym.: Trichogrammatidae) para o controle de Diatraea saccharalis (Fabr., 1794) (Lep.: Pyralidae). 141p. (Tese mestrado), ESALQ, USP, Piracicaba.

LOPES JRS; PARRA JRP. 1991. Efeito da idade de ovos do hospedeiro natural e alternativo no desenvolvimento e parasitismo de duas espécies de Trichogramma. Revista de Agricultura 66: 221-244.

MITCHELL ER; HU GY; OKINE JS. 1998. Diamondback moth (Lepidoptera: Plutellidae) infestation and parasitism by Diadegma insalure (Hymenoptera: Ichneumonidae) in collards and adjacent cabbage fields. Florida Entomologist 80: 54-61.

NAVARANJAN AV. 1979. Influence of host age on parasitism by Trichogramma australicum and T. japonicum (Himenoptera: Trichogrammatidae). Journal of Applied Entomology 87: 277-281.

OLIVEIRA HN. 1997. Aspectos biológicos e taxa de parasitismo de Trichogramma maxacalii em ovos de Anagasta kuehniella. 39p.(Tese mestrado), UFV, Viçosa.

OLIVEIRA HN; PRATISSOLI D; ZANUNCIO JC; SERRÃO JE. 2003. Influência da idade dos ovos de Oxydia vesulia no parasitismo de Trichogramma maxacalii. Pesquisa Agropecuária Brasileira 38: 551-554. 
PAK GA. 1988. Selection of Trichogramma for inundative biological control: a study of behavioural variations among strains and species of an egg-parasite genus. Wageningen: Landbouwuniversiteit the Wageningen, p.224.

PARRA JRP. 1997. Técnicas de criação de Anagasta kuehniella, hospedeiro alternativo para produção de Trichogramma. In: PARRA JRP; ZUCCHI RA. (Ed.) Trichogramma e o controle aplicado. Piracicaba: FEALQ, cap.4, p.121-150

PEREIRA FF; BARROS R; PRATISSOLI D 2004. Desempenho de Trichogramma pretiosum Riley e T. exiguum Pinto \& Platner (Hymenoptera: Trichogrammatidae) submetidos a diferentes densidades de ovos de Plutella xylostella (Lepidoptera: Plutellidae). Revista Ciência Rural 34: 1669-1674.

PEREZ CJ; SHELTON AM; ROUSH RT. 1997. Managing diamondbackmoth (Lepidoptera: Plutellidae) resistance to foliar applications of Bacillus thuringiensis: testing strategies in field cages. Journal of Economic Entomology 90: 1463-1470.
PRATISSOLI D; PEREIRA FF; BARROS R; PARRA JRP; PEREIRA CLT. 2004a Parasitismo de Trichogramma pretiosum em ovos da traça-das-crucíferas sob diferentes temperaturas. Horticultura Brasileira 22: 754 757.

PRATISSOLI D; HOLTZAM; GONÇALVES JR; VIANNA UR; BELLINI LL. 2004b. Efeito da ausência de hospedeiro e de alimento sobre aspectos biológicos de Trichogramma pretiosum Riley (Hymenoptera: Trichogrammatidae). Acta Scientiarum 26: 281-286.

SCHMIDT JM; SMITH JJB. 1987. The measurement of exposed host volume by the parasitoid wasp Trichogramma minutum and effects of wasp size. Canadian Journal of Zoology 65: 2837-2845.

TABONE E; PINTUREAU B; PIZZOL J; MICHEL F; BARNAY O; TABONE E. 1999. Aptitude de 17 souches de Trichogramma a parasiter lateigne dês cruciferes Plutella xylostella L. em laboratoire (Lep.: Yponomeutidae). Annales de la Société Entomologique de France 35: 427-433.
VAN DIJKEN MJ; KOLE M; VAN LENTEREN JC; BRAND AM. 1986. Host-preference studies with Trichogramma evanescens Westwood (Hym.: Trichogrammatidae) for Mamestra brasicae, Pieris brasicae and Pieris rapae. Journal of Applied Entomology 101:6485

VASQUEZ LA; SHELTON AM; HOFFMANN MP; ROUSH RT. 1997. Laboratory evaluation of commercial Trichogrammatid products for potential use against Plutella xylostella (L.) (Lepidoptera: Plutellidae). Biological Control 9: 143-148

WUHRER BG; HASSAN SA. 1993. Selection of effective species/strains of Trichogramma (Hym.: Trichogrammatidae) to control the diamondback moth Plutella xylostella L. (Lep.: Plutelidae). Journal Applied Entomologic 116: 80-89.

ZUCCHI RA; MONTEIRO RC. 1997. O gênero Trichogramma na América do Sul. In: PARRA JRP; ZUCCHI RA. (Ed.) Trichogramma e o controle aplicado. Piracicaba: FEALQ, cap.2, p.41-66. 